\title{
Reflexiones sobre la relación entre la comunidad académica de defensa y el CDS
}

\section{Reflections on the relationship between the academic defense community and the CDS}

Marina Vitelli*

\section{INTRODUCCIÓN}

Un proceso de construcción de gobernanza regional está ocurriendo en la dimensión de la defensa en América del Sur, a partir de la creación del Consejo Sudamericano de Defensa (CDS). Partiendo del entendimiento de que los factores de unidad superan a los aun existentes elementos de discordia, los países de la UNASUR se propusieron sumar la dimensión de la defensa al proyecto de diálogo político de América del Sur, constituyendo un organismo de consulta, cooperación y coordinación en materia de defensa.

Tal como ocurre con toda iniciativa de integración regional, la terminología usada para comunicar objetivos, acciones y logros suele aparecer teñida del tono fuertemente optimista propio del discurso político. Teniendo en cuenta la aspereza de la realidad internacional, de los enfrentamientos entre intereses nacionales, los celos sobre las respectivas soberanías y las cuentas pendientes en materia de institucionalización de la integración en América del Sur, ciertamente el lenguaje del CDS puede sonar excesivamente optimista: un organismo que propone consolidar a Sudamérica como zona de paz y construir una identidad regional en defensa.

\footnotetext{
* Profesora Jefe de Trabajos Prácticos da Universidad Nacional de Rosario, Facultad de Ciencia Política y Relaciones Internacionales. Becaria postdoctoral del Consejo Nacional de Investigaciones Científicas y Tecnológicas (CONICET). E-mail: marinagvitelli@gmail.com.
} 
No fueron pocos, sin embargo, quienes desafiaron la tradicional perspectiva sombría respecto a las relaciones entre los Estados y se animaron a hablar de identidades colectivas, comunidades de seguridad y zonas de paz. Algunos buscaron las raíces de las instituciones regionales de seguridad en el cálculo estratégico frente a una amenaza común, otros en las normas y valores compartidos. Por nuestra parte, en el marco de este trabajo destacamos aquellos estudios que otorgan un lugar de relevancia a los agentes e iniciativas vinculadas a la investigación y la reflexión como variable coadyuvante para la cooperación en defensa. Partimos del supuesto planteado en trabajos anteriores respecto a que el CDS constituye un esquema de cooperación regional en defensa en construcción con una especificidad: antes de conformar una alianza operacional y un mecanismo de seguridad colectiva, se propuso iniciar un proceso gradual pero profundo de consensuar un pensamiento común de defensa (Vitelli, 2013). Esta tarea, elemento propio de una identidad colectiva, es realizada a partir de la organización de seminarios, cursos para funcionarios e intercambios académicos, actividades que tuvieron distinto nivel de concreción, como analizaremos más adelante.

Continuando con el tema y enfoque de nuestra tesis doctoral y motivados por la creación de la Revista Brasileira de Estudos de Defesa nos interrogamos en este trabajo sobre el vínculo existente entre el CDS y la comunidad regional de estudios de defensa. Comenzamos por reseñar algunos de los desarrollos teóricos mas importantes sobre la relación entre conocimiento experto y cooperación regional en defensa, para luego rastrear las instituciones claves de la comunidad regional de estudios sobre esta cuestión, mencionando algunos de sus vínculos con las políticas de defensa nacionales y con la cooperación regional en defensa. A continuación evaluamos de forma crítica el tipo de relación que el CDS plantea con esta comunidad, subrayando la insuficiencia en materia de interacción con ella, aun cuando parte importante de la naturaleza de este órgano se relaciona con la práctica misma de la comunidad académica: el diálogo y la discusión sobre enfoques y conceptos. Para finalizar, expresamos algunas reflexiones sobre el potencial de los aportes de los expertos civiles a la cooperación regional en defensa.

\section{EXPERTOS Y FUNCIONARIOS EN LA TEORÍA}

La política de defensa tiene una característica fundamental que la dife- 
rencia de otras políticas públicas: la existencia de una corporación específica, con fuerte sentido de la verticalidad y con gran espíritu de cuerpo. Lo que es más, las fuerzas armadas tiene el control del instrumento militar: constituyen una corporación armada. Ciertamente, el hecho de que los estudios tiendan a acentuar en los análisis el peso de las fuerzas armadas en la formulación de las políticas de defensa refleja este dato de la realidad, tornando a la defensa en un ámbito poco propicio para la influencia de los expertos civiles.

Esta observación es pertinente para estudios de distintas áreas geográficas pero lo es aún más en relación a aquellos trabajos que investigan sobre las políticas de defensa de los países latinoamericanos. Sin dudas, el pasado autoritario con graves casos de tutelas militares sobre democracias débiles y golpes de estados con dictaduras castrenses ha dejado fuertes improntas sobre los gobiernos actuales, variables imposibles de desconocer cuando se analizan políticas de defensa en la región. A pesar de esto, y teniendo en cuenta los avances relativos alcanzados en materia de democratización y lo sostenido en relación al CDS, vale la pena poner de relieve los desarrollos que desde la teoría de las Relaciones Internacionales han subrayado la participación de actores no tradicionales en la formulación de las políticas de defensa, entre ellos los expertos.

Ciertamente, el concepto que primero viene a la mente cuando se habla de la interacción entre saber y política es el de comunidades epistémicas, aquellas redes de expertos que logran influir sobre la política exterior de los Estados, en particular en áreas sometidas a la cooperación internacional. Desde este enfoque, Emanuel Adler demostró que las ideas teóricas y prácticas de la comunidad epistémica del control de armas estratégicas que se conformó en Estados Unidos se convirtieron en expectativas políticas, se difundieron hacia la Unión Soviética y fueron luego reflejadas en el Tratado Antibalístico de 1972 sobre control de armas (Adler, 1992). Mientras que en un principio el concepto estuvo teñido de un fuerte matiz científico-técnico, otros hemos señalado su valor analítico para el estudio de la influencia de expertos con saberes más blandos, destacando también las especificidades que lo alejan del concepto más genérico de comunidad académica (Davis Cross, 2013; Vitelli, 2015).

Por su parte, Risse demostró que algunos de los drásticos cambios de política exterior implementados por el gobierno de Gorbachov ocurrieron en parte importante debido a la influencia de una red transnacional de políticos y académicos occidentales y de Europa del Este. Concretamente, 
la interacción y el diálogo entre la arriba mencionada comunidad científica norteamericana sobre control de armas estratégicas, los investigadores de la paz de Europa del Este y los políticos de la social-democracia alemana y el partido laborista holandés junto a la Internacional Socialista hicieron posible el desarrollo conceptual que transformó el concepto estratégico de la disuasión hacia la perspectiva de la seguridad común y la defensa no-ofensiva, fundamentos del posterior modelo de la seguridad cooperativa. Aquellas instituciones luego establecieron contacto con científicos soviéticos, analistas políticos de instituciones científicas y académicos especializados en política exterior. Estos new thinkers transmitieron a los líderes soviéticos los conceptos que se habían derivado del célebre Informe Plame, los cuales planteaban la necesidad de reducir las capacidades y la postura ofensiva de las fuerzas armadas soviéticas, así como de comprometerse con amplios regímenes de control y reducción de armas estratégicas (Risse-Kappen, 1995: 219).

Retornando a lo trabajado por Adler, este continuó estudiando las comunidades epistémicas, término que reconoció como una subcategoría de otro concepto más amplio: las comunidades de prácticas (Adler, 2005: 13). Desde un enfoque similar, aunque utilizando esta nueva categoría para estudiar a esas redes de individuos que se forman en las relaciones transnacionales regulares, Adler (2008) analizó el devenir de la OTAN y argumentó que la ampliación exitosa ocurrida durante los noventa fue posible gracias a la acción de una comunidad de prácticas de la seguridad cooperativa originada durante el proceso de Helsinki. Si bien el foco del análisis está puesto en las interacciones entre los funcionarios del organismo, Adler reconoció que los expertos académicos también jugaron un papel relevante en la difusión del concepto de seguridad cooperativa.

El argumento teórico que Adler desarrolló a partir de estos estudios constituye el corazón de la variante comunitarista del constructivismo en Relaciones Internacionales, la cual involucra a los expertos en el análisis de la política internacional. Esta formulación busca "comprender los fundamentos de los procesos de gobernanza regional desde la importancia que tienen en los mismos la construcción de significados comunes y los procesos de evolución cognitiva que ocurren al interior de las comunidades transnacionales de funcionarios y expertos" (Adler, 2005: 21). Así, una comunidad de prácticas implica la existencia de un grupo de personas dedicadas regularmente a un asunto, la configuración de un dominio de entendimientos consensuados, y una práctica compartida. 
Desde esta perspectiva teórica se entiende que parte importante de la internalización de las ideas sobre cómo cooperar para resolver problemas internacionales se da a través de la participación periódica de los agentes estatales en determinadas prácticas comunes (Adler, 2005: 21). El supuesto subyacente es que "los intereses de los Estados se desarrollaron primariamente porque en primer lugar una comunidad de prácticas intentó influir sobre el esquema conceptual utilizado para pensar sobre los intereses" (Adler, 2008: 200). Una vez que esto ocurre y aquellos significados se institucionalizan, pasan también a expresarse en las racionalidades y las rutinas organizacionales del organismo internacional, tal como ocurrió con el modelo de la seguridad cooperativa en la OTAN.

\section{LA EXPERIENCIA REGIONAL: LA COMUNIDAD ACADÉMICA DE DEFENSA}

Otro elemento que suma a la relevancia de pensar sobre el vínculo entre los funcionarios oficiales de las instancias de cooperación regional en defensa y los expertos en la materia es la existencia en América Latina de un conjunto de instituciones e iniciativas que conformaron la comunidad regional de estudios de defensa. Los especialistas que construyeron esa comunidad ejercieron en distinta medida algún tipo de impacto sobre las políticas de defensa de los estados.

Como parte de nuestras investigaciones hemos analizado la influencia que sobre la política de defensa argentina tuvo una comunidad epistémica, formada a partir de un núcleo de asesores legislativos, políticos y expertos académicos (Vitelli, 2015). La institución que conformaron - SER en el 2000 - fue la encargada de llevar adelante una de las instancias regionales de diálogo sobre defensa más sostenidas en el tiempo: el Seminario Permanente Hacia las Fuerzas Armadas del año 2000. Entre 1990 y 1997 se realizaron un total de 65 encuentros, los cuales solían comenzar con la exposición de un experto que funcionaba como disparador para la discusión del grupo.

El seminario no sólo incluyó invitados argentinos. De hecho, su mayor riqueza probablemente fue la interacción con miembros de otras importantes instituciones de expertos de la región, tales como el Núcleo de Estudios Estratégicos de la UNICAMP, el Instituto Brasileiro de Assuntos Estratégicos (IBAE), el Centro de Estudios Estratégicos de la Secretaría de Asuntos Estratégicos de la presidencia (CEE-SAE) y FLACSO- Chile. $\mathrm{El}$ impacto que sobre las políticas de los gobiernos tuvieron las actividades de investigación y reflexión llevadas adelante por FLACSO-Chile y SER 
en el 2000 encuentra un fuerte indicador en el hecho de que ambas instituciones hayan sido convocadas para la construcción en 1995 del Comité Permanente de Seguridad entre Chile y Argentina (COMPERSEG), el esquema de diálogo bilateral en materia de cooperación en defensa.

De gran relevancia para la comunidad regional de estudios de defensa fue el rol jugado por la mencionada institución chilena, tanto en calidad de editora de la Revista Fuerzas Armadas y Sociedad -i mportante foro de debate sobre la cuestión en los años noventa- como por su asociación con el think tank norteamericano Woodrow Wilson International Center for Scholars como co-organizadores del Programa Paz y Seguridad en las Américas. En el marco de este proyecto fueron celebrados seminarios y talleres de reflexión y debate, resultado de los cuales se publicaron libros que plasmaron las principales ideas relativas a la reforma de las fuerzas armadas latinoamericanas y el modelo de seguridad cooperativa como alternativa para la región.

Ciertamente, parte importante de las iniciativas de investigación y reflexión sobre defensa y seguridad de esta época estuvieron teñidas por la influencia de la agenda norteamericana para el hemisferio. En este marco, tanto los miembros de SER en el 2000 como los expertos brasileños y los chilenos - entre otros - fueron asiduos participantes de las iniciativas del Center for Hemispheric Defense Studies (CHDS) ${ }^{1}$, creado como resultado de la II Conferencia de Ministros de Defensa de las Américas en 1996 alrededor del programa de investigación y acción del concepto de seguridad cooperativa impulsado por el secretario de Defensa de Estados Unidos, William Perry.

Los lazos entre los miembros de la comunidad regional de estudios de defensa continuaron estrechándose durante los primeros años del siglo XXI. En 2001 comenzó a tomar forma lo que pocos años después sería la Red de Seguridad y Defensa de América Latina (RESDAL). Junto con importantes proyectos de investigación que reúnen a expertos de las tres Américas, esta institución con sede en Buenos Aires es la responsable de la edición del Atlas Comparativo de la Defensa, importante obra de consulta de la comunidad regional de expertos. De similar manera, aunque con mayor énfasis en la agenda de las nuevas amenazas, el Programa de Cooperación en Seguridad Regional de la Friedrich Ebert Siftung, también realiza actividades de reflexión sobre seguridad y defensa, incluida la temática de 
la cooperación, publicando su Anuario de la seguridad regional.

A pesar de ser una institución nacional, la creación de la ABED en 2006 puede entenderse como uno de los acontecimientos más relevantes de la comunidad regional de estudios de defensa. En principio, por la consabida importancia de Brasil para la región, el país de mayores dimensiones, recursos y ambiciones de presencia global, elementos que suman incentivos a las investigaciones y reflexiones sobre la vinculación entre política de defensa y política exterior. De igual manera, la constitución de la ABED representa la maduración de un conjunto de instituciones universitarias que vienen desarrollando programas de investigación sobre defensa y seguridad desde el retorno de la democracia. Ciertamente, la cooperación regional en defensa constituye una de las principales temáticas tratadas por los miembros de la ABED en las distintas instancias institucionales de diálogo y reflexión. Finalmente, es innegable el dinamismo que ha adquirido esta institución, demostrado por la realización de ocho encuentros anuales y otros tantos de alcance regional, y por la constitución ahora de su Revista.

A lo largo de casi tres décadas, actores políticos y académicos de la región han establecido vínculos transnacionales y se han involucrado en intercambios de ideas y conceptos, cuyo impacto sobre las políticas de defensa puede ser difícil de medir, pero no por ello despreciable. Innegablemente, los eventos de discusión y las publicaciones que resultaron de los mismos fueron conformando una verdadera agenda de investigación, que incluyó, entre otros temas, a las medidas de fomento de la confianza, las relaciones civiles-militares, el control civil sobre las fuerzas armadas, las problemáticas de seguridad en clave de nuevas amenazas y los modelos subregionales de cooperación en defensa. Ahora bien, esa red transnacional de expertos existe aun sólo en términos analíticos ya que sus lazos son informales, esporádicos y no logran vincular a todos los miembros. Este legado actualmente desaprovechado podría ser recuperado poniéndolo en relación con el énfasis que el CDS intenta colocar en la formación y capacitación para la defensa y su vínculo con la conformación de una identidad sudamericana de defensa. 
RBED, v. 2, no 1, jan./jun. 2015

\section{EL CDS Y LOS ESTUDIOS DE DEFENSA EN LA CONSTRUCCIÓN DE UNA IDENTIDAD ESTRATÉGICA REGIONAL}

Retomando el supuesto sobre la especificidad del modelo de seguridad regional que representa el CDS, decíamos que este se planteó una serie de propósitos relacionados con la construcción de una identidad estratégica regional, tales como "avanzar gradualmente en el análisis y discusión de los elementos comunes de una visión conjunta en materia de defensa”, y "avanzar en la construcción de una visión compartida respecto de las tareas de la defensa” (Vitelli, 2013). De similar manera, su Centro de Estudios Estratégicos de Defensa (CEED-CDS), la instancia de producción de investigación a efectos del asesoramiento al CDS, se ha propuesto como misión "la generación de conocimiento y difusión de un pensamiento estratégico suramericano en materia de defensa y seguridad regional e internacional” (CDS, 2010).

Estos y otros elementos constituyen indicadores que llevan a pensar que el tipo de cooperación regional en defensa que se propone el CDS se basa fundamentalmente en la creación conjunta de significados a través de un trabajo conjunto de diálogo, en lugar de otro tipo de cooperación, como la gestión de crisis que amenazan la paz y la seguridad, tal como es el caso del Consejo de Seguridad de Naciones Unidas. A partir de esto, entendemos que lo realizado por el CDS se centra en la construcción de una identidad estratégica regional a partir de la puesta en marcha de una comunidad de prácticas del tipo de las estudiadas por Adler, esto es: un proceso de gobernanza regional que consta de un grupo de funcionarios oficiales, un dominio de entendimientos consensuados, y una práctica compartida consistente en instancias de investigación, discusión y negociación a partir de las cuales se originan esos entendimientos.

En lo que hace a este tipo de prácticas subrayamos la organización por parte del CDS de más de una docena de seminarios y talleres sobre temas como el valor estratégico de los recursos naturales, la modernización de los ministerios de defensa, el rol de las fuerzas armadas frente a los desastres naturales y los conceptos y enfoques sobre defensa. Otro importante ejemplo de actividad de aprendizaje conjunto es el Curso Avanzado de Defensa Sudamericano (CAD-Sul), que cada año, desde 2012, reúne a funcionarios de defensa -tanto militares como civiles- durante dos meses en la Escuela 
Superior de Guerra de Brasil para impartir contenidos sobre caracterización de los países de la UNASUR, organismos internacionales, geopolítica y geoestrategia, seguridad y defensa, industrias de material de defensa y el CDS. De similar manera el CDS realizó tres ediciones del Programa Suramericano de Formación de Civiles en Defensa, entre 2012 y 2014, con instancias virtuales y otras presenciales en Buenos Aires.

Sin dudas, la iniciativa más representativa de este pilar del modelo de seguridad regional que el CDS apunta a construir es la creación de la Escuela Sudamericana de Defensa. La ESUDE - proyecto surgido en 2012, cuyos estatuto y reglamento fueron aprobados en noviembre del 2014- fue concebida como un centro de altos estudios y de articulación de redes entre las iniciativas nacionales de los Estados miembros, para la formación y capacitación de civiles y militares en materia de defensa y seguridad regional.

Ahora bien, a pesar de que el CDS, en particular a partir de su CEED, ha apostado a la creación de instancias de investigación y reflexión sobre temas de seguridad y defensa, hasta el momento no ha establecido iniciativas de articulación con la importante comunidad regional de estudios de defensa arriba descripta, conformada por instituciones académicas de distinto tipo, constituida fundamentalmente por expertos civiles. Así, mientras que los dos primeros planes de acción del CDS incluían bajo el eje Formación y Capacitación la confección de una base de datos con información sobre las instituciones militares y de los centros de formación de especialistas civiles en defensa, así como el establecimiento de programas de intercambio docente y estudiantil, homologación, evaluación y acreditación de estudios, reconocimiento de títulos y becas entre las instituciones existentes, en materias de defensa, ambas tareas no volvieron a ser incluidas en los planes de acción y tampoco se informó el grado de avance obtenido?

Podría pensarse que una vez iniciada la actividad del CEED este punto hubiera pasado a su órbita, puesto que el artículo X de su Estatuto establece como una de las funciones del mismo el establecimiento, a través de los ministerios de defensa de "relaciones institucionales y una red de intercambios con los centros de estudios estratégicos nacionales que conforman el CDS y con centros extra regionales que este determine pertinente" (CDS, 2010). Pero en la sección de los centros nacionales contrapartes de su sitio web - designados por los ministerios de defensa para colaborar con las 
investigaciones del CEED - aparecen listadas solamente instituciones de tipo gubernamental y con un acentuado sesgo de pertenencia militar.

De igual manera, tampoco tuvo continuidad una actividad estipulada en el primer plan de acción del CDS: el I Encuentro Sudamericano de Estudios Estratégicos. No sólo subrayamos el hecho de que este evento celebrado en noviembre de 2009 tuvo una sola edición, sino también que se realizó en el marco del IX Encuentro Nacional de Estudios Estratégicos de Brasil, lo que llevó a una sobrerrepresentación de expertos de ese país y el correspondiente déficit de participación de estudiosos del resto de la región, en particular de las instituciones arriba mencionadas.

Por último, cabe mencionar una propuesta académica incluida en el primer Informe de Gestión del CDS sobre la cual no existe información que corrobore su ejecución ni su continuidad. Nos referimos al Proyecto de formación de cuadros académicos para la consolidación de la comunidad epistémica de la defensa de América del Sur, presentado por la coordinación de la línea de concentración Paz, Defensa y Seguridad del Programa de posgrado interinstitucional San Tiago Dantas, cuya propuesta consistía en becar conjuntamente a estudiantes de posgrado de los países miembros del CDS, sean funcionarios gubernamentales o expertos académicos, para realizar el curso en Brasil.

\section{COMENTARIOS DE CIERRE}

A partir de lo constatado en el apartado anterior, entendemos que el CDS apuesta a la concertación política en defensa a partir de la generación de enfoques y conceptos comunes, involucrando a los funcionarios nacionales en diversas iniciativas de aprendizaje. A pesar de que en los documentos oficiales se expresa la voluntad de incluir también a las instituciones académicas vinculadas a la defensa, hasta el momento los expertos civiles han sido los grandes ausentes en estas instancias, espacio mayormente ocupado por las instituciones académicas militares. Aun más paradójico es el hecho de que existe una comunidad regional de estudios de defensa con muchos años de experiencia, aunque con débiles lazos institucionales.

En función de lo expuesto hasta aquí realizamos un llamamiento a una mayor interacción entre las iniciativas del CDS y el trabajo de investigación de los expertos civiles pertenecientes a las instituciones académicas. 
Esta vinculación redundaría en mayores chances de éxito de la construcción de la identidad regional en defensa. De igual manera, un incremento de los análisis académicos, posibilitado por una mayor apertura de las instancias oficiales del CDS, significaría la multiplicación de estudios desde una perspectiva crítica sobre un área de cuestiones que tradicionalmente ha tendido a escapar de la transparencia y la accountability.

Por último, lejos de sostener que el saber práctico de los funcionarios ministeriales tiene menos valor que el conocimiento académico de los investigadores destacamos la especificidad de este último: la articulación con un campo disciplinar, sea este la Ciencia Política, las Relaciones Internacionales o la Sociología. El conocimiento académico implica una interrelación entre los desafíos de la vida real con las discusiones teóricas que marcan la evolución de una disciplina. Esto es importante porque la teoría no sólo es una herramienta para comprender la realidad: también permite entender por qué las relaciones entre Estados llegaron a ser lo que son y cuáles son las posibilidades de superar desigualdades y conflictos. Es esta faceta crítica y normativa la que jugó un rol fundamental en los procesos de cambio pacífico hacia esquemas de cooperación reseñadas en el primer apartado de este trabajo, un antecedente auspicioso para el proyecto de la integración regional sudamericana. 
RBED, v. 2, no 1, jan./jun. 2015

\section{NOTAS}

1. Entre 2001 y 2003 el CHDS celebró tres seminarios titulados Research and Education in Defense and Security Studies (REDES), en los cuales participaron gran parte de los expertos de la comunidad regional. En la actualidad el Perry Center realiza anualmente diversos cursos destinado a militares, funcionarios políticos de defensa y seguridad y académicos provenientes de las Américas. 2. Ecuador construyó un portal en el cual se listan las instituciones y sus cursos, pero este no aparece en el sitio web del CDS - el cual pasa largos periodos sin estar disponible- ni en el del CEED. De todas maneras, el portal incluye una abrumadora mayoría de instituciones militares. En la sección Brasil, el único centro no militar incluido es la PUC-SP y llama la atención que la ABED no está incluida. Disponible en: http://unasur.midena.gob.ec , consultado en marzo de 2015. 


\section{REFLEXIONES SOBRE LA RELACIÓN ENTRE LA COMUNIDAD ACADÉMICA DE DEFENSA Y EL CDS}

\section{RESUMEN}

El artículo pone en valor el rol de los expertos académicos en las iniciativas de cooperación regional. Si bien el CDS valoriza las instancias de investigación y reflexión sobre conceptos y enfoques de defensa, no incluye de forma apropiada a la comunidad académica de estudios de defensa existente en Sudamérica.

Palabras claves: CDS, comunidad académica, cooperación regional, defensa.

\section{ABSTRACT}

While the South American Defense Council takes seriously the importance of research and discussion on defense policy concepts and approaches, it still does not appropriately include the extensive South American academic community of defense studies. The article highlights the role of academic experts in regional cooperation.

Keywords: South American Defense Council, academic community, regional cooperation, defense.

\section{REFERENCIAS}

Adler, E. (1992) "The Emergence of Cooperation : National Epistemic Communities and the International." International Organization, 16 (1), pp: 101145 .

Adler, E. (2005) Communitarian International Relations. The Epistemic Foundations of International Relations. Routledge.

Adler, E. (2008) "The Spread of Security Communities: Communities of Practice, Self-Restraint, an NATO's Post Cold War Transformation." European Journal of International Relations, 14 (2), pp: 195-230.

CDS. (2010, mayo 5) Estatuto del Centro de Estudios Estratégicos del CDS. Recuperado a partir de www.ceedcds.org.ar.

Davis Cross, M. K. (2013) "Rethinking epistemic communities twenty years 
RBED, v. 2, no 1 , jan./jun. 2015

later." Review of International Studies, 39 (1), 137-160.

Risse-Kappen, T. (1995) "Ideas do not float freely: transnational coalitions, domestic structures, and the end of the cold war." En International Relations Theory and the end of the cold war. Columbia University Press, pp: 187-222.

Vitelli, Marina. (2013). “Teoría y práctica de las identidades colectivas en las Relaciones Internacionales. El caso del Consejo de Defensa Sudamericano”. Reflexión Política 15 (30), pp: 54-64.

- (2015) Argentina, Brasil y la defensa en América del Sur. Las

identidades estratégicas y la seguridad regional. Tesis de doctorado, Facultad de Ciencia Política y Relaciones Internacionales de la Universidad Nacional de Ciencia Política. 